\title{
Discoid lupus erythematosus progressing in active systemic lupus erythematosus and non-Hodgkin's lymphoma: case report
}

\begin{abstract}
We report on a 54-year-old woman with long-lasting discoid lupus erythematosus (DLE) who presented at our department with discoid rash, fever, moderate cervical lymphadenopathy, extreme fatigue. Before that time, her DLE symptoms had been controlled with NSAIDs, and low-dose prednisone. Three weeks before presentation, she experienced fevers ranging from 38.0 to $39.0^{\circ} \mathrm{C}$, but she had no specific symptoms that suggested local infection. Physical examination, multiple blood cultures, and other laboratory investigations were performed to make the point of the situation. Abnormal laboratory parameters in cell blood count together with creatinine alteration and the appearance of antibodies to double-stranded DNA were crucial to make a diagnosis of an active Systemic Lupus Erythematosus (SLE). Important elevations of triglycerides and lactate dehydrogenase ( $\mathrm{LDH}$ ) found in the analysis, oriented us to further examine the patient toward a hematological condition. The biopsy from the axillary lymphonodes showed high grade diffuse large B-cell (CD20+, Ki-67+, Bcl$2+)$ non-Hodgkin's lymphoma. Through this case report we present a rare event when DLE progresses to SLE associated to non-Hodgkin's lymphoma. In these cases it is important a differential diagnosis that should take into account the possibility of active SLE, infection, and malignancy.
\end{abstract}

Keywords: malignancy, discoid lupus erythematosus, non-Hodgkin's lymphoma, systemic lupus erythematosus
Volume 2 Issue 5 - 2018

\author{
Pecani A,' Zoto A,' Bushati T, ${ }^{2}$ Backa T' \\ 'Rheumatology Unit, Department of Internal Medicine, \\ University Hospital Center "Mother Teresa" Tirana, Albania \\ 2Laboratory of Pathology, University Hospital Center "Mother \\ Teresa" Tirana, Albania
}

Correspondence: Pecani A, Rheumatology Unit, Department of Internal Medicine, University Hospital Center "Mother Teresa” Tirana,Albania, Email arbipekani@gmail.com

Received: April 18, 2018 | Published: October 09, 2018
Abbreviations: DLE, discoid lupus erythematosus; SLE, systemic lupus erythematosus; LDH, lactate dehydrogenase; ALP, alkaline phosphatase; GGT, gamma-glutamyl transferase; ANA, antinuclear antibody; CT, computed tomography; NHL, nonHodgkin's lymphoma

\section{Case presentation}

A 54-year-old Albanian woman with a 30-year history of discoid lupus erythematosus (DLE), presented at our department with discoid rash, fever, night sweating, moderate cervical lymphadenopathy, extreme fatigue accompanied by a rapid and recent weight loose. The diagnosis of DLE had been made years ago based on the presence of discoid rash and photosensitivity. Other criteria for diagnosing SLE were not met at all her previous visits of follow up. She was under treatment with prednisone $10 \mathrm{mg}$ daily and ibuprofen 400 $\mathrm{mg}$ as needed, to control her DLE symptoms. For a period of three weeks, the patient had fevers ranging from 38.0 to $39.0{ }^{\circ} \mathrm{C}$, treated by her family doctor with antibiotic therapy but with short term results in lowering the fever. At the moment of presentation she had no symptoms or complains that might indicate a local infection; increasing fatigue was her most prominent complain. The difficulties in breathing, cough and sputum disappeared after using Seretide twice a day and Ventolin once a day as the patient had been diagnosed even with Bronchial Asthma 2 years ago. On physical examination it was noticed that she had a temperature of $38.9^{\circ} \mathrm{C}$ with moderate cervical lymphadenopathy and splenomegaly. An infectious etiology of fever was ruled out through multiple blood cultures and urine cultures that resulted negative for microbial growth. Laboratory investigation revealed a hemoglobin concentration of $10,3 \mathrm{~g} / \mathrm{dl}$, with leucopenia $\left(2100 / \mathrm{mm}^{3}\right)$ and relative thrombocytopenia $\left(9000 / \mathrm{mm}^{3}\right)$. There was evidence of an increased erythrocyte sedimentation rate of $80 \mathrm{~mm} / \mathrm{h}$ together with a high serum C-reactive protein $(\mathrm{CRP}=219 \mathrm{mg} / \mathrm{dl})$, a serum lactate dehydrogenase level of $805 \mathrm{U} / 1$ (normal range 122-220 $\mathrm{U} / 1$ ), aspartate-aminotransferase and alanine-aminotransferase levels slightly elevated to normal levels while gamma-glutamyl transferase (GGT) and alkaline phosphatase (ALP) were elevated to twice the normal levels (180 IU/1 and $350 \mathrm{IU} / 1$, respectively).

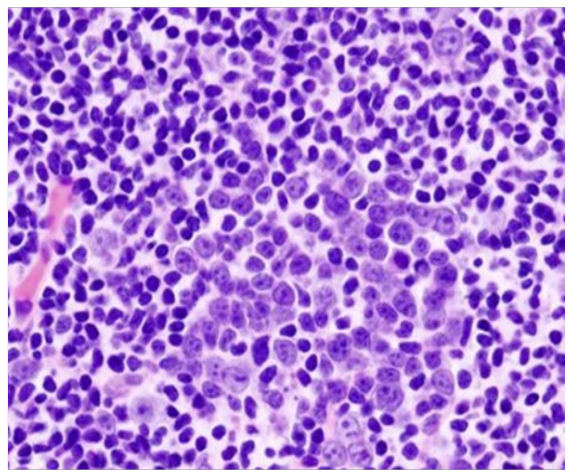

Figure I Histopathology of high grade diffuse large B-cell non-Hodgkin's lymphoma with a starry sky pattern (representative image).

All these abnormal laboratory parameters were not present in all the previous routine visits of the patient at our ambulatory service. Immunofluorescence techniques revealed a recent antinuclear antibody (ANA) present at a high titer with a homogeneous pattern, followed by a low positive antibody titter to double-stranded DNA. To note, both these immunological findings has not been present before. Regarding the complement levels in the serum, there was a $\mathrm{C} 3$ of $141 \mathrm{mg} / \mathrm{dL}$ and $\mathrm{C} 4$ of $38.3 \mathrm{mg} / \mathrm{dL}$ accompanied by a low titer of $\mathrm{IgG}$ 
and $\operatorname{IgM}(618$ and 14.10 respectively) and a negative test for ENA (extractable nuclear antigen). Serologic tests for Salmonella Typhi O and $\mathrm{H}$, brucellosis, hepatitis $\mathrm{B}+\mathrm{C}$ virus, cytomegalovirus and $\mathrm{HIV} 1+2$ were negative. Despite the immunosuppressive therapy applied since the patient was admitted to the hospital, her overall health condition did not improve. The laboratory parameters started to get more altered, now including even creatinine (up to $3.4 \mathrm{mg} / \mathrm{dL}$ ) and uremia (up to $161 \mathrm{mg} / \mathrm{dL}$ ) levels as well as total and direct bilirubin level. Hypoalbuminemia occurred as well. Meanwhile, in her $24 \mathrm{~h}$ urine collection, there was evidence of proteinuria $(0.86 \mathrm{~g} / \mathrm{L})$, cellular casts. Three day after the patient was hospitalized; axillary's and inguinal bilateral lymphadenopathy appeared rapidly increasing in their size. The lymph nodules had a diameter approximately from $4-5 \mathrm{~cm}$ with no tenderness in palpation. In computed tomography (CT) of chest and abdomen, additional mediastinal, supraclavicular and para-aortal lymphadenopathy were clearly noted. Bone-marrow biopsy showed pancytopenia but couldn't give more detailed information regarding the disease. In these conditions, consulting even a hematology and infectious-disease specialists it was performed a biopsy of the axillaries lympho nodes. After hematoxylin and eosin stain the histopathological data showed high grade diffuse large B-cell (CD20+, Ki-67+, Bcl-2+, CKAE1/AE3 -) non-Hodgkin's lymphoma (NHL) with a starry sky pattern as showed in Figure 1. The patient was diagnosed as stage IV diffuse large B-cell lymphoma (DLBCL) concomitant to DLE that has recently progressed in active SLE (SLEDAI $2 \mathrm{~K}=6$ ). The general health condition of the patient were not stable despite the therapy applied thus it was not possible to start the chemotherapy. Seven days after, the patient deceased.

\section{Discussion}

SLE is an autoimmune disorder in which the immune system attacks inappropriately the healthy tissue of the organism leading like this to chronic inflammation. According to The American College of Rheumatology (ACR), there are 11 classification criteria for the clinical diagnosis of SLE (Table 1) from which the patient should fulfill at least 4 criteria in order to be considered as having the disease. ${ }^{1}$ Discoid lupus erythematosus (DLE) is a chronic photosensitive, localized or widespread, skin eruption usually affecting females of all ethnic groups between the age of 20-45 years old. ${ }^{2}$ DLE may occur together with SLE or without systemic involvement. In this case the patients will present only with skin involvement. ${ }^{3}$ In this case report, up to now, the patient had never fulfilled the ACR criteria for SLE. She was 24 years old at the time when a DLE diagnosis was meeting and for over 30 years, the only clinical finding has been the discoid rash by ruling out a diagnosis of SLE. For the purpose of identifying patients for clinical studies, an individual is considered to have SLE if at least 4 of the 11 criteria are present, serially or simultaneously. Most of the patients with DLE have a favorable prognosis considering mortality although morbidity should always be taken in consideration. Malignant degeneration in DLE patients is uncommon, however significant changes of the cutaneous lesions in patients with longlasting disease should be considered for biopsy prompt biopsy. ${ }^{4}$

In this case, the patient presented with a known history of DLE and new symptoms of fever, lymphadenopathy and extreme fatigue. Of note, fever that lasts for several weeks without a specific cause identified, despite extensive continuous examinations, constitutes a fever of unknown origin (FUO). In patients presenting with FUO, the physician should take a detailed history of the disease accompanied with a careful physical examinations in order to discover the etiology of the fever. Besides infective diseases, the most common causes of FUO include malignancy and autoimmune rheumatic diseases like SLE. ${ }^{5}$ The new - onset of anemia with leucopenia and relative thrombocytopenia accompanied by a positive serology (ANA, antiAND ds) and alteration of renal parameters (creatinine, uremia) resulting in excessive proteinuria and cellular casts, all features that have never been present before, indicate a recent active SLE in our patient. Data from the literature support the fact that DLE progress in SLE in about $10 \%$ of the patients, ${ }^{6}$ as it was in this case. On the other hand, lymphadenopathy is a common finding in almost $67 \%$ of SLE patients with active disease, ${ }^{7}$ but that should normally disappear as soon as the immunosuppressive therapy starts otherwise it should be considered as a signal for further testing and multidisciplinary team consultation consultations with a hematologist, oncologist, and infectologist. ${ }^{8}$ Fever of long duration, without sepsis, and accompanied by lymphadenopathy and organomegaly together with laboratory signs anemia and elevated lactate dehydrogenase levels found in our patient, were indicative of a hematological malignancy. A biopsy and tissue diagnosis are essential to confirm a diagnosis of hematological malignancy. Bone-marrow biopsy showed pancytopenia in our patient while the histopathological report of the lympho nodes biopsy confirmed a stage IV diffuse large B-cell lymphoma.

Table I The American college of rheumatology revised criteria for the classification of systemic lupus erythematous

Malar rash (fixed eruption over the cheeks)

\section{Discoid rash}

Photosensitivity (appearance/worsening of rash after light exposure)

Oral or nasopharyngeal ulcers

Nonerosive inflammatory polyarthritis (peripheral joints)

Pleuritis or pericarditis

Persistent proteinuria, or cellular casts in urine

Seizures or psychosis in the absence of drugs or metabolic disturbances Hematological disorder (hemolytic anemia, leucopenia, lymphopenia, or thrombocytopenia)

Positive test for antinuclear antibodies

Positive test for antibodies to double-stranded DNA, Smith antigen, or antiphospholipid (including lupus anticoagulant or false-positive syphilis test).

Pancytopenia has been considered as a predictive hematological finding in the development of NHL. ${ }^{9}$ The risk of hematological malignancies is around three times higher in patients with lupus compared to the general population (standarderized incidence ratio $2.75,95 \%$ CI 2.13 - 3.49). In particular, this increased risk is associated to NHL (standardized incidence ratio 3.64, 95\% CI 2.63-4.93). ${ }^{10}$ According to the medical literature, most of this risk is attributed to the dysregulation of the immune system and the use of immunosuppressive therapies. ${ }^{11}$ In the study of Bernatsky et al., the use of cyclophosphamide together with high cumulative dose corticosteroids have been considered as predictors of increased lymphoma risk is SLE patient ${ }^{9}$ although some other studies showed no significant association in this regard. ${ }^{12,13} \mathrm{~A}$ unique finding about this case, is the fact that the patient had never been under conventional or biologic DMARDs since there was no evidence of systemic involvement for over 30 years. In this regard, the patient has been taking only prednisone from time to time and Hydroxychloroquine for a short period at the moment that the diagnosis of DLE was 
established. Thus, in this case the role of immunosuppressive therapy as a possible trigger of the NHL is not that much significant and can be ruled out while the immune system dysregulation remain as the crucial factor in this regard. The most common NHL in SLE patients is the DLBCL type constituting about $50-60 \%$ of the cases. ${ }^{12,13}$

An important role in the biological pathway of lymphoma, may be attributed to the over proliferation of lymphocytes and the fact that patients with SLE are also more vulnerable to viruses than the general population, which can increase their cancer risk. ${ }^{12,13}$ As here reported, our patient has been treated for three weeks with antibiotics due to an infection that her family doctor has diagnosed and that may have served as a trigger for SLE and NHL as well. The exact mechanism of lymphagenesis in SLE is not fully elucidated. Gene expression analysis has put in evidence two different subgroups of DLBCL with different pathophysiological mechanisms and different therapeutically response. In this regard the most important types of NHL are: "germinal center B-cell-like DLBCL" originating from the germinal centre of B cells and "activated B-cell-like DLBCL" deriving from postgerminal center of B cells. Of note, the activated B-celllike DLBCL has the worst prognosis. ${ }^{14}$ Furthermore, serum lactate dehydrogenase levels are considered as a predictive prognostic factor in lymphoma. ${ }^{15} \mathrm{~A}$ high level of LDH is associated to an aggressive lymphoma, as in our case. DLBCL arising in patients with a history of SLE are mostly of non-GCB subtype, and this has potential implications on therapy and prognosis. ${ }^{14}$ In the light of this facts, we may explain the irresponsiveness of the disease to the treatment as well as the rapid progression in this case.

\section{Conclusion}

Lupus is an autoimmune disease associated with increased frequency of neoplasia and lymphoma. Although the presenting symptoms of lymphoma might vary, fever, lymphadenopathy and fatigue are common. The differential diagnosis should take into account the possibility of active SLE, infection, and malignancy. Diffuse large B-cell lymphoma is the most common histological type in lupus, and the most common subtype of aggressive NHL. An awareness of the association of Hodgkin's disease with DLE/SLE and the modes of presentation will help in the early diagnosis and clinical management of such patients.

\section{Author contributions}

All authors participated in the completion of this work. They participated in the implementation of the clinical case and the proceeding discussion.

\section{Acknowledgements}

None.

\section{Conflict of interest}

The author declares there is no conflict of interest.

\section{References}

1. Tsokos GC. Systemic lupus erythematosus. The New England Journal of Medicine. 2011;365(22):2110-2121.

2. Okon LG, Werth VP. Cutaneous Lupus Erythematosus: Diagnosis and treatment. Best Pract Res Clin Rheumatol. 2013;27(3):391-404.

3. Grönhagen CM, Fored CM, Granath F, et al. Cutaneous lupus erythematosus and the association with systemic lupus erythematosus: a population-based cohort of 1088 patients in Sweden. Br J Dermatol. 2011;164(6):1335-41.

4. Tao J, Zhang X, Guo N, et al. Squamous cell carcinoma complicating discoid lupus erythematosus in Chinese patients: review of the literature, 1964-2010. J Am Acad Dermatol. 2012;66(4):695-696.

5. Gaeta GB, Fusco FM, Nardiello S. Fever of unknown origin: a systematic review of the literature for 1995-2004. Nucl Med Commun. 2006;27(3):205-11.

6. Chand AY, Werth PV. Treatment of Cutaneous Lupus. Curr Rheumatol Resp. 2011;13(4):300-307.

7. Brown JR, Skarin AT. Clinical mimics of lymphoma. The Oncologist. 2004;9(4):406-416.

8. Gillmore R, Sin WY. Systemic lupus erythematosus mimicking lymphoma: the relevance of the clinical background in interpreting imaging studies. BMJ Case Reports. 2014;pii: bcr2013201802.

9. Bernatsky S, Ramsey-Goldman R, Joseph L, et al. Lymphoma risk in systemic lupus: effects of disease activity versus treatment. Ann Rheum Dis. 2014;73(1):138-142.

10. Bernatsky S, Boivin JF, Joseph L, et al. An international cohort study of cancer in systemic lupus erythematosus. Arthritis Rheum. 2005;52(5):14811490 .

11. Bernatsky S, Ramsey-Goldman R, Labrecque J, et al. Cancer risk in systemic lupus: an updated international multi-centre cohort study. Journal of Autoimmunity. 2013;42:130-135.

12. Kang KY, Kim HO, Yoon HS, et al. Incidence of cancer among female patients with systemic lupus erythematosus in Korea. Clinical Rheumatology. 2010;29(4):381-388

13. Löfström B, Backlin C, Sundström C, et al. A closer look at non-Hodgkin's lymphoma cases in a national Swedish systemic lupus erythematosus cohort: a nested case-control study. Ann Rheum Dis. 2007;66(12):16271632 .

14. Hassan U, Mushtaq S, Mamoon N, et al. Prognostic sub-grouping of diffuse large B-cell lymphomas into germinal centre and post germinal centre groups by immunohistochemistry after 6 cycles of chemotherapy. Asian Pacific Journal of Cancer Prevention. 2012;13(4):1341-1347.

15. Fisher RI. Overview of non-Hodgkin's lymphoma: biology, staging, and treatment. Semin Oncol. 2003;30(4):3-9. 